\title{
Rebar Presence in the Structures using Electrical Property of Concrete: A Review
}

\author{
Amala Joy \\ Post Graduate student, Department of Civil Engineering \\ Sree Narayana Gurukulam College of Engineering \\ Ernakulam, kerala, India
}

\author{
Jeena Mathew \\ Department of Civil Engineering \\ Sree Narayana Gurukulam College of Engineering \\ Ernakulam, India
}

\begin{abstract}
The structural reliability of existing structures will become a more frequent task for engineers in the future due to the increasing age of existing infrastructure. The Presence of cracks in concrete structure has been one of the major causes of structural failure. To ensure the structural reliability of existing structures, identifying the presence of rebar is essential. Early prediction of rebar presence will help as to reduce the cost in terms of rehabilitation works. Electrical resistivity of concrete can be used to detect the presences of rebar, which may help to predict the reliability of concrete structure in terms of reinforcement cover, quantity, quality etc.. Wenner Probe technique can be used to measure the resistivity of concrete from the surface in seconds without the need for coring. In this paper, the correlation between electrical resistivity embedded rebar presences on concrete electrical resistivity is discussed. Also this paper reviews factors affecting presences of rebar and electrical resistivity.
\end{abstract} rebar

Keywords-Electrical resistivity; Presences of embedded

\section{INTRODUCTION}

Concrete is one of the most frequently used building material worldwide. It is an important construction material used extensively in civil infrastructure that includes building water systems, highways, bridges, dams, and numerous other structural applications. Taking versatility of concrete to means its 'adaptability to a wide variety of purposes', it is critical to guarantee that concrete that is used in structures has the highest possible quality and specified properties. To ensure the structural reliability of existing structures, identifying the presence of rebar is essential. Early prediction of rebar presences will help as to reduce the cost in terms of rehabilitation works. Non Destructive test is a method of testing existing concrete structures to assess the strength and durability of concrete structure. Active infrared thermography with microwave excitation and the eddy current technique are most commonly used for detection of deeply located rebar. But the instrument used for Non Destructive test is costly and difficult to handle. So we usually doesn't prefer this kind of instruments in India. Therefore in order to overcome this problem, electricity resistivity of concrete is used to detect rebar presence.

Electrical resistivity of concrete is a property used to assure the quality of concrete by detecting the presences of cracks and rebar, which help to predict the reliability of concrete structure. Electrical resistivity $(\rho)$ of a material is defined as its capability to withstand the transfer of ions subjected to an electrical field. Concrete resistivity $\rho$ ranges widely from $10^{1}$ to $10^{6} \Omega \mathrm{m}$, depending on mainly the moisture content and the material of the concrete. There are a number of techniques to measure concrete resistivity. However, the four-probe Wenner probe technique is the most commonly used technique because of its speed, simplicity, and practicality. It can be used to measure the resistivity of concrete from the surface in seconds without any intrusive process such as coring.

In this paper, the correlation between electrical resistivity and presence of rebar, is discussed which could be used as a tool to predict the service life of the structure.

\section{INFLUENCING PARAMETERS ON ELECTRICAL RESISTIVITY MEASUREMENTS DUE TO THE PRESENCE OF REBAR}

A number of researchers have been done on the effect of embedded rebar presence on concrete electrical resistivity through experimental investigation. Researchers have been investigating the factors in concrete that may affect electrical resistivity measurements. A number of factors such as diameter of rebar, cover thickness of concrete, the orientation of the reinforcement with respect to the probe could affect electrical resistivity measurements.

Millard [8] and Gower and Millard [6], studied the effect of rebar diameter and spacing, as well as the effect of concrete cover thickness on electrical resistivity measurements using a four-point Wenner probe. Millard's experimental investigation consisted of tanks filled with conductive solution and steel rebar placed parallel to one another within the solution. Probe measurements were taken in between rebar and parallel to rebar at different distances away from the rebar. The main influencing factor was found to be the distance taken away from the rebar, while rebar diameter was not found to be significant in its disturbance. Reduced rebar spacing was found to increase measurement errors. The experimental investigation lacked the use of actual concrete and did not investigate different probe configurations with respect to the rebar or rebar mesh.

Carino [2], provided a summary of the literature for nondestructive test methods when assessing reinforced concrete and stated how reinforcing bars affect electrical resistivity measurements in that the apparent resistivity was lower than the true resistivity value. Carino also restated how concrete cover thickness and the effect of embedded rebar had a strong correlation in that with smaller cover thickness will create for greater error. It was also suggested to take readings midway between two rebar, and suggested that when concrete 
cover thickness and rebar spacing were small, that a correction factor was possible when the diameter and location of the reinforcement was known. The correction factor was not explained in detail. No experimental research was conducted, and no recommendations were provided for the presence of rebar mesh.

Sengul and Gjorv [10], conducted an experimental investigation to understand the effects of cover thickness, electrode spacing, embedded rebar, probe measurement directions relative to the embedded rebar, and probe measurement distance away from the embedded rebar. Fourpoint Wenner probes, each with different electrode spacing, were used to conduct the electrical resistivity testing for reinforced concrete test specimens as well as for field measurements on concrete structures. Five different probe configurations with respect to embedded rebar were considered, where four of the five configurations were parallel to rebar, and the fifth was perpendicular to rebar as seen in Fig 1 .

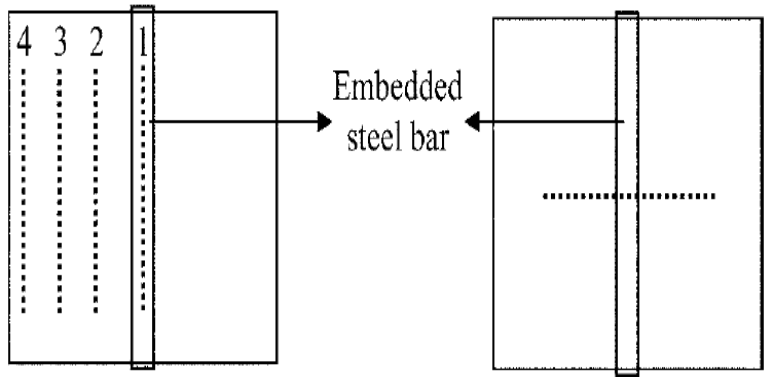

Fig 1. Five Wenner probe with respect to embedded rebar tested by Sengul and Gjorv [10]

It was concluded that embedded rebar and electrode spacing affect observed resistivity of concrete. For larger electrode spacing, parallel measurements with respect to embedded rebar significantly reduced observed resistivity, whereas when measurements were made perpendicular to rebar, there were no significant effects to observed resistivity. . Sengul and Gjorv [10], suggest that all measurements should be taken as far away as possible from embedded rebar, and if dense reinforcement makes this impossible, electrode spacing should be kept small relative to cover depth. Similar to Gowers and Millar's [6], study, only one rebar was placed within the reinforced concrete block and rebar mesh was not simulated for the laboratory experimental investigation. Rather than sizable reinforced concrete slabs, the measurements were conducted on small specimens which possibly contributed errors to the study due to edge effects. Also concluded that measurements taken perpendicular to the rebar were not significantly influenced by the rebar. Since only one embedded rebar was considered in these studies, and the slab size was relatively small, this conclusion is difficult to extrapolate to real cases.

Salehi et al. [7], investigated the effect of the presence of rebar mesh in concrete on electrical resistivity measurements conducted with a four-point Wenner probe. The effects of different concrete thicknesses, rebar diameter, rebar mesh densities and probe configurations with respect to the rebar mesh were studied. It was concluded that the smallest error will result while setting up the probe parallel to the top rebar within the rebar mesh and perpendicular to bottommost rebar during measurements taken as illustrated in Fig 2. It was also found that the observed resistivity decreased once the rebar mesh densities increased and the rebar diameter effect on concrete resistivity measurements can be neglected. There was no experimental investigation to confirm the conclusions based on the numerical study results, but it was one of the few investigations that includes rebar mesh and probe configuration with respect to rebar in its assessment of the effects of reinforcement to electrical resistivity measurements.

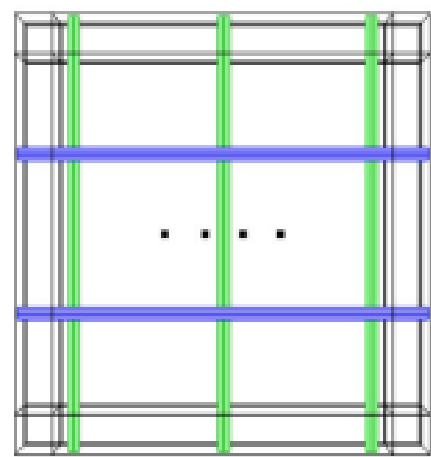

Fig 2. Probe with respect to rebar mesh suggested to reduce electrical resistivity measurement error by Salehi et al. [7]

Chen et al. [3], experimentally investigated the influences of specimen shape and size, electrode spacing, and cover thickness on electrical resistivity measurements made with a four-point Wenner probe. It was suggested that a correction factor should be applied to resistivity measurements corresponding with the ratio of specimen length to electrode spacing as well as the ratio of specimen diameter to electrode spacing for cylindrical concrete specimens. For prismatic specimens, that the effects by the probe spacing can be ignored when the spacing is larger than $40 \mathrm{~mm}$. However, the resistivity values increased with less electrode spacing. For application of Wenner probe method, the important role of electrode spacing should be definitely considered during electrical resistivity measurements as it will affect the obtained results.

Weyder and Gehlen [11], studied the effects on Wenner probe electrical resistivity measurements on reinforced concrete blocks in which rebar was spaced parallel to one another. It was found that orienting the probe orthogonally with respect to the embedded rebar, errors on electrical resistivity measurements were reduced. This study lacked the investigation of the effect of rebar mesh and only studied where rebar segments were parallel. When Wenner probe was placed perpendicular to the rebars, the errors associated with the presence of reinforcement were reduced. However it is noted that, in practical cases reinforced concrete structures that rebar is in mesh form spanning both transversely and longitudinally within the concrete, which then may have different influences on measured electrical resistivity than the effect of parallel rebar spanning in only one direction.

Polder et al. [9], provided practical general guidelines for taking resistivity measurements on concrete by revisiting and summarizing existing literature. In the absence of research on resistivity measurements over rebar meshes, it was suggested 
that resistivity measurements should be taken in diagonal orientations from the mesh. Taking several measurements in this arrangement and averaging the results were also recommended. However, there are no experimental or theoretical studies to support this suggested scheme. Moreover, depending on the spacing between rebars and electrodes of the Wenner probe, it is not possible to have all four electrodes inside the rectangular unit created by the crossing rebars at all times. Thus, in some scenarios it is possible to have one or more of the electrodes located on top of the rebars and this arrangement might significantly affect resistivity measurements. Also concluded that the electrical current may travel through the concrete volume with approximately the same depth as that of the electrode spacing. Hence, as the probe spacing increases, the current flows deeper inside the concrete volume and when the electrical current reaches the surface of the rebar, the current is transported through the reinforcement and, thus, results in lower resistivity observation.

Presuel-Moreno [4],conducted numerical and experimental investigations involving the effect of the orientation and location of Wenner probe resistivity measurements with respect to embedded rebar. The investigation also involved the effect of the number of embedded rebar on measurements. This research is examples of the few studies taking into account rebar mesh rather than studying the effect of a single rebar within concrete, as well as the angle of the Wenner probe with respect to rebar (not just parallel and perpendicular configurations). Five different orientations were studied for the presence of rebar mesh, and six different orientations were studied for the presence of a single rebar. From the experimental and numerical results, it was observed that when more than one rebar was present, as in rebar mesh, observed resistivity was significantly reduced. This showed the importance that investigations should be conducted considering rebar mesh when assessing effects on electrical resistivity measurements using a four-point Wenner probe. However, only one rebar mesh density was considered in the study, where smaller rebar to rebar spacing may have different effects on electrical resistivity measurements. Also, most of the probe configurations were investigated within the square created by the four rebar segments. Further investigation in probe configuration with respect to rebar within rebar mesh was not conducted. Different rebar mesh densities and concrete cover thicknesses were also not considered in the study.

Garzon et al. [1], experimental study, small scaled cylindrical and prismatic specimens were casted. As polarization will happen due to double layer at the steel and concrete interface acting as a resistancecapacitor, resistivity measurements taken directly above rebar will result in errors. Hence, a rebar factor was suggested to be applied to the obtained resistivity results. In addition, modified Wenner equations are recommended for various geometric parameters. Only reinforced cylindrical and prismatic specimens were included in the experimental setup without considering a reinforced slab. However, in a numerical study, a slab with embedded rebar was considered. The experimental investigation lacked in using concrete mixture instead of a mortar mixture which is not exactly representative of realworld cases and may lead to more errors. Furthermore, the proposed rebar factor may not be applicable to a large concrete slab with multiple rebar because their experimental conclusions are based on laboratory testing.

Lim et al. [12], also studied the effects of cover depth, electrode spacing, rebar diameter, and the resistivity of concrete and reinforcement in the numerical model. However, only one probe configuration taken right above and parallel to rebar was considered. It was suggested to apply a geometric effect rate that ranges from 0 to 1 in order to estimate the reinforcement geometry impact and this rate is derived utilizing a resistivity estimation model. The geometric effect rate was also validated through the experimental investigation for onsite measurements. Based on experimental findings, it was stated that the geometric effect rate decreased with increasing concrete cover thickness and increased with increasing rebar diameter and increasing electrode spacing. Again, using mortar mixture, only one single rebar, and a single probe configuration with respect to rebar is not completely representative of realworld conditions. An error to resistivity measurements may also be introduced while the epoxy coating on the mortar specimens was used in this study due to a barrier between the electrodes and mortar surface.

The last and recent study in this category belongs to Sanchez et al. [5], who numerically proposed a modified 4point Wenner method based on the experimental data, deployed on a bridge over the River Danube in Romania. In this study, a "rebar factor," $f b$, was introduced through the modified version of Wenner method to measure the resistivity in reinforced concrete structures with thin concrete covers. Effect of rebar presence on concrete electrical resistivity measurements also can be found in detail in similar studies by Gowers and Millard.

\section{III.SUMMERY AND CONCLUSION}

By the extensive evaluation of the above literature review, this paper identifies several factors which might have influence on the electrical resistivity of concrete. Effect of each parameter is briefly summarized below.

In the most of studies, when there is an embedded rebar in the concrete, the electrical current field is distorted, and thus errors can result in the electrical resistivity measurements. To minimize this effect, it is suggested to place all electrodes perpendicular to the embedded rebar on the concrete surface and take at least five measurements, each a few millimetres in distance from one another.

Also showed that using smaller electrode spacing would decrease measurement deviation from the actual resistivity of concrete when measurements are taken over rebar mesh. Likewise, presence of rebars parallel to the Wenner probe was found to have the most adverse effect on the performance of the probe. Previously, the location of rebars perpendicular to the probe was assumed to have no significant impact on resistivity measurement. However, with rebar mesh, location of perpendicular rebars play a significant role such that measurements could vary as much as $20 \%$ with the change in location of just perpendicular rebar with respect to the probe. As reported by Millard [10], variation of rebar diameter was found to have some influence on measurements; however, this effect was negligible. In an existing structure neither the cover 
thickness nor rebar spacing would be under the control of the examiner. Perhaps choosing different electrode spacing for the probe is possible but location or orientation of the probe can be easily managed on site. As much as $30 \%$ increase in accuracy of concrete resistivity measurement can be achieved just by selecting appropriate orientation or location for the Wenner probe. In general, variations observed by change in either cover thickness or rebar spacing would affect this distance. Theoretically, closer the rebars to current electrodes, the easier the current fluxes in concrete can reach them. Hence, more portion of the current tends to pass through the rebars instead of concrete. However, effect of rebar presence on the resistivity measurements is well-understood. But just a few studies could be found to identify the rebar presence effect and more field investigations are still needed in this area.

\section{REFERENCES}

[1] A.J. Garzon "Modification of four point method to measure the concrete electrical resistivity in presence of reinforcing bars", ELSEVIER 53 (2014) 249-257.

[2] Carino, N.J "Nondestructive techniques to investigate corrosion status in concrete structures", Journal of performance of constructed facilities, 1999. 13(3): p. 96-106.
[3] Chen, C.-T., J.-J. Chang, and W.-c. Yeih,'The effects of specimen parameters on the resistivity of concrete. Construction and Building Materials," 2014. 71(0): p. 35-43.

[4] F. Presuel-Moreno "Numerical modeling of the effects of rebar presence and/or multilayered concrete resistivity on the apparent resistivity measured via the Wenner method", ELSEVIER 48 (2013) 16-25.

[5] J. Sanchez, C. Andrade, J. Torres, N. Rebolledo, and J. Fullea, "Determination of reinforced concrete durability with on-site resistivity measurements," Materials and Structures, vol. 50, no.1, 2017.

[6] K. R. Gowers and S. G. Millard, "Measurement of concrete resistivity for assessment of corrosion severity of steel using wenner technique," ACI Materials Journal, vol. 96, no. 5, 1999.

[7] M. Salehi, Numerical investigation of the role of embedded reinforcement mesh on electrical resistivity measurements of concrete using the Wenner probe technique, Carleton University, Ottawa, Canada, 2013.

[8] S.G. Millard, "Reinforced concrete resistivity measurement techniques," Institution of Civil Engineers, Vol. 91, pp. 71-88, 1991.

[9] R. B. Polder, "Test methods for on site measurement of resistivity of concrete," Construction and Building Materials, vol. 15, no. 2-3, pp. 125-131, 2001.

[10] Sengul, O, \& Gjorv, O. E "Effect of embedded steel on electrical resistivity measurements on concrete structures", ACI Materials Journal, (2009), Vo1. 6, Pp. 11-18.

[11] Weydert R, Gehlen C "Electrolytic resistivity of cover concrete: relevance, measurement and interpretation", NRC Research Press, (1999), Vol. 1, Pp .409-419.

[12] Y.-C. Lim, T. Noguchi, and C.-G. Cho, "A quantitative analysis of thegeometric effects of reinforcement in concrete resistivity measurement above reinforcement," Construction and Building Materials, vol. 83, pp. 189-193, 2015. 